\title{
Peroxisome Proliferator-Activated Receptor alpha Induction of Uncoupling Protein 2 Protects Against Acetaminophen-Induced Liver Toxicity
}

\author{
Andrew D. Patterson, ${ }^{1,2}$ Yatrik M. Shah, ${ }^{1,3}$ Tsutomu Matsubara, ${ }^{1}$ Kristopher W. Krausz, ${ }^{1}$ \\ and Frank J. Gonzalez ${ }^{1}$
}

Acetaminophen (APAP) overdose causes acute liver failure in humans and rodents due in part to the destruction of mitochondria as a result of increased oxidative stress followed by hepatocellular necrosis. Activation of the peroxisome proliferator-activated receptor alpha $(P P A R \alpha)$, a member of the nuclear receptor superfamily that controls the expression of genes encoding peroxisomal and mitochondrial fatty acid $\beta$-oxidation enzymes, with the experimental ligand $W_{y}-14,643$ or the clinically used fibrate drug fenofibrate, fully protects mice from APAP-induced hepatotoxicity. PPAR $\alpha$-humanized mice were also protected, whereas Ppara-null mice were not, thus indicating that the protection extends to human PPAR $\alpha$ and is PPAR $\alpha$-dependent. This protection is due in part to induction of the PPAR $\alpha$ target gene encoding mitochondrial uncoupling protein 2 (UCP2). Forced overexpression of UCP2 protected wildtype mice against APAP-induced hepatotoxicity in the absence of PPAR $\alpha$ activation. Ucp2-null mice, however, were sensitive to APAP-induced hepatotoxicity despite activation of PPAR $\alpha$ with $W y-14,643$. Protection against hepatotoxicity by UCP2-induction through activation of PPAR $\alpha$ is associated with decreased APAPinduced c-jun and c-fos expression, decreased phosphorylation of JNK and c-jun, lower mitochondrial $\mathrm{H}_{2} \mathrm{O}_{2}$ levels, increased mitochondrial glutathione in liver, and decreased levels of circulating fatty acyl-carnitines. These studies indicate that the PPAR $\alpha$ target gene UCP2 protects against elevated reactive oxygen species generated during drug-induced hepatotoxicity and suggest that induction of UCP2 may also be a general mechanism for protection of mitochondria during fatty acid $\beta$-oxidation. (НерATOLOGY 2012;56:281-290)

$\mathrm{P}$ eroxisome proliferator-activated receptor alpha $(\operatorname{PPAR} \alpha)$, a member of the nuclear receptor superfamily, controls the expression of a battery

Abbreviations: ALT, alanine aminotransferase; APAP, acetaminophen; $A S T$ aspartate aminotransferase; GSH, glutathione; NAPQI, N-acetyl-pbenzoquinone imine; PPAR $\alpha$, peroxisome proliferator-activated receptor alpha; $R O S$, reactive oxygen species; UCP2, uncoupling protein 2.

From the ${ }^{1}$ Laboratory of Metabolism, National Cancer Institute, National Institutes of Health, Bethesda, MD; ${ }^{2}$ Center for Molecular Toxicology and Carcinogenesis, Department of Veterinary and Biomedical Sciences, Pennsylvania State University, University Park, PA; ${ }^{3}$ Department of Molecular and Integrative Physiology and Internal Medicine, Division of Gastroenterology, University of Michigan School of Medicine, Ann Arbor, MI.

Received July 21, 2011; accepted January 24, 2012.

Address reprint requests to: Frank J. Gonzalez, Laboratory of Metabolism, National Cancer Institute, National Institutes of Health, Bethesda, MD 20814.E-mail: gonzalef@mail.nih.gov; fax:301-496-8419.

Copyright $($ () 2012 by the American Association for the Study of Liver Diseases. View this article online at wileyonlinelibrary.com.

DOI 10.1002/hep. 25645

Potential conflict of interest: Nothing to report.

Additional Supporting Information may be found in the online version of this article. of genes involved in lipid homeostasis including those encoding peroxisomal and mitochondrial enzymes that carry out fatty acid catabolism. PPAR $\alpha$ is mainly expressed in organs that are critical in fatty acid catabolism, such as liver, heart, and kidney. ${ }^{1-3}$ Perhaps the most critical role of PPAR $\alpha$ is to modulate hepatic fatty acid catabolism. In untreated mice, PPAR $\alpha$ controls constitutive expression of mitochondrial fatty acid $\beta$-oxidation enzymes. ${ }^{4}$ During periods of starvation in mice PPAR $\alpha$ is activated, resulting in induction of both mitochondrial and peroxisomal fatty acid catabolism. ${ }^{5}$ Notably, in the course of spontaneous and ligand-induced activation of fatty acid catabolism excess $\mathrm{H}_{2} \mathrm{O}_{2}$ is produced as a byproduct of induction of peroxisomal acyl-CoA oxidase. Reactive oxygen species (ROS) are also produced during mitochondrial fatty acid $\beta$-oxidation. Although this increase in $\mathrm{H}_{2} \mathrm{O}_{2}$ is dealt with in part by catalase, glutathione peroxidase, and manganese superoxide dismutase, the cellular responses to ROS are saturated upon the massive 
activation of fatty acid catabolism that occurs after ligand activation of PPAR $\alpha$. Consequently, increased PPAR $\alpha$ activity during accelerated fatty acid catabolism is associated with increased expression of free-radical scavengers such as catalase and $\mathrm{Cu} / \mathrm{Zn}$ dismutase ${ }^{6}$ and mitochondrial uncoupling proteins (UCPs) that may serve to reduce mitochondrial ROS levels. ${ }^{7,8}$ Both direct and indirect effects suggest that $\operatorname{PPAR} \alpha$ may serve a protective role to combat the deleterious side effects of fatty acid catabolism, thus preserving, in particular, mitochondrial function.

Increased ROS levels are frequently associated with hepatotoxicity produced by overdose of drugs such as acetaminophen (APAP). APAP, the most common nonprescription analgesic used for pain relief and antipyresis, is a representative compound that causes liver toxicity upon overdose and is a significant public health concern due to occasional overdose in children and adults. ${ }^{9,10}$ A reactive quinone metabolite, $N$-acetyl-p-benzoquinone imine (NAPQI), generated by cytochrome P450-catalyzed oxidation, triggers hepatic toxicity by covalently binding with nucleophilic macromolecules and/or by elevating ROS leading to apoptosis and cell necrosis. ${ }^{11}$

Interestingly, a connection between $\operatorname{PPAR} \alpha$ and APAP toxicity was established when it was discovered that pretreatment with clofibrate, a PPAR $\alpha$ activator, protected mice against APAP-induced hepatotoxicity $^{12,13}$ and that this protection was $\operatorname{PPAR} \alpha$-dependent. ${ }^{14}$ Furthermore, it was recently reported that toxic doses of APAP inhibit fatty acid $\beta$-oxidation and that these effects were significantly reduced in mice lacking the major enzyme responsible for the bioactivation of APAP, CYP2E1, due, in part, to enhanced and persistent activation of PPAR $\alpha$ and its target genes. ${ }^{15}$ Wildtype mice treated with APAP, however, showed suppressed PPAR $\alpha$ activity. Thus, PPAR $\alpha$ may function to protect mitochondria from ROS that occurs during APAP metabolism and as a natural consequence during fatty acid catabolism. In the present study the protective effects of PPAR $\alpha$ activation during APAPinduced hepatotoxicity were further investigated and a role for the PPAR $\alpha$ target gene UCP2 in mediating these protective effects explored.

\section{Materials and Methods}

Animals. Wildtype (C57Bl/6J) and ucp2-null $\left(\mathrm{B} 6.129-U_{c p} 2^{\text {tm1Low1}} / \mathrm{J}\right)$ mice were obtained from the Jackson Laboratories (Bar Harbor, ME). Ppara-null mice and wildtype counterparts on the $129 / S_{\mathrm{S}}$ background were described previously. ${ }^{16}$ The PPAR $\alpha$ - humanized mouse was described previously. ${ }^{17}$ All animal experiments were carried out in accordance with the Institute of Laboratory Animal Resources guidelines and approved by the National Cancer Institute Animal Care and Use Committee.

Acetaminophen Experiments. Groups of 6 to 8week-old male mice were fed Wy-14,643 (0.1\%) diet for 24 hours before an intraperitoneal injection of APAP $(400 \mathrm{mg} / \mathrm{kg})$ dissolved in saline. All mice were euthanized by $\mathrm{CO}_{2}$ asphyxiation 2 hours, 6 hours, or 24 hours after the APAP dose. Livers were harvested and stored at $-80^{\circ} \mathrm{C}$ before analysis. To assess liver damage, tissue was briefly washed with phosphate-buffered saline (PBS) and fixed in 10\% neutral buffered formalin. Necrosis was scored by hematoxylin and eosin (H\&E) staining. APAP-induced liver injury was determined by measuring aspartate aminotransferase (AST) and alanine aminotransferase (ALT) catalytic activities in serum using a commercial AST or ALT assay kit (Catachem, Bridgeport, CT). Reduced glutathione (GSH) levels in liver were measured by a glutathione assay kit (Sigma-Aldrich, St. Louis, MO) and liver hydrogen peroxide $\left(\mathrm{H}_{2} \mathrm{O}_{2}\right)$ levels were determined by use of the Peroxidetect kit (Sigma-Aldrich).

RNA Analysis. Dye-coupled complementary DNAs (cDNAs) were purified with a MiniElute PCR purification kit (Qiagen) and hybridized to an Agilent $44 \mathrm{~K}$ mouse 60-mer oligo microarray (Agilent Technologies, Santa Clara, CA). The data were processed and analyzed by Genespring GX software (Agilent Technologies). Significance was determined as $>10$-fold to the wildtype control samples. Specific messenger RNA (mRNA) levels were determined by quantitative realtime polymerase chain reaction (qPCR). RNA was extracted using TRIzol reagent (Invitrogen, Carlsbad, CA) and qPCR performed using cDNA generated from $1 \mu \mathrm{g}$ of total RNA with SuperScriptII Reverse Transcriptase (Invitrogen). Primers for qPCR were designed using the Primer Express software (Applied Biosystems, Foster City, CA). qPCR reactions were carried out using the SYBR Green PCRmaster mix (SuperArray, Frederick, MD) and an ABI Prism 7900HT Sequence Detection System (Applied Biosystems). Quantitation was carried out using the comparative cycle threshold (CT) method and results were normalized to mouse $\beta$-actin.

Generation and Infection of the Adenovirus Constructs Expressing Recombinant UCP2. Rat UCP2 adenovirus was obtained from the Gene Transfer Vector Core (University of Iowa). ${ }^{18}$ For in vivo infection of recombinant adenoviruses, 6 to 8-weekold wildtype mice were intravenously injected in the 
tail vein with $1.2 \times 10^{10}$ infection units, in a total volume of $400 \mu \mathrm{L}$, of recombinant adenoviruses expressing UCP2 or with an adenovirus expressing Cre recombinase used as a control. Two days later, mice were administered APAP and the mice killed after 6 hours or 24 hours.

Western Blot Analysis. Liver whole cell or mitochondrial extracts were prepared and subjected to electrophoresis on sodium dodecyl sulfate-polyacrylamide gel electrophoresis (SDS-PAGE). Membranes were incubated with antibodies against CYP2E1, total JNK, p-JNK (Cell Signaling Technologies, Danvers, MA), or UCP2 (Santa Cruz Biotechnology, Santa Cruz, CA). JNK kinase assays were performed using the nonradioactive SAPK/JNK kinase assay kit (Cell Signaling Technologies) according to the manufacturer's instructions.

Quantitation of APAP Metabolites and Serum Palmitoylcarnitine. APAP serum metabolites (APAP, APAP-NAC, APAP-glucuronide, APAP-CYS) were monitored as described. ${ }^{19}$ For serum palmitoylcarnitine, deproteinated serum samples from wildtype mice were analyzed by use of an API2000 triplequadrupole mass spectrometer (Applied Biosystems). Debrisoquine was used as internal standard. Samples were injected into a high-performance liquid chromatography (HPLC) system (PerkinElmer, Waltham, MA) using a Luna C18 column (Phenomenex, Torrance, CA; $50 \times$ $2.1 \mathrm{~mm}$ i.d.). The flow rate through the column at ambient temperature was $0.3 \mathrm{~mL} / \mathrm{min}$ with a gradient (methanol:water:acetonitrile, containing $0.1 \%$ formic acid) from 5:60:35 to 5:5:90 in a 6-minute run. The column was equilibrated for 1.5 minutes before each injection. The mass spectrometer was operated in the turbo ion spray mode with positive ion detection; the turbo ion spray temperature was maintained at $350^{\circ} \mathrm{C}$ and a voltage of $4.5 \mathrm{kV}$ was applied to the sprayer needle. Nitrogen was used as the turbo ion spray and nebulizing gas. Detection and quantification were performed using the multiple reactions monitoring mode, with $\mathrm{m} / \mathrm{z}$ 400.3/85.0 for palmitoylcarnitine and $m / z$ 176.1/134.2 for debrisoquine. Using Analyst software (Applied Biosystems), serum palmitoylcarnitine concentrations were determined by calculating the ratio between the peak area of palmitoylcarnitine and the peak area of debrisoquine and fitting with a calibration curve with a linear range from $10 \mathrm{nM}$ to $1 \mu \mathrm{M}(r=0.99)$.

Statistical Analysis. Statistical analysis was performed using GraphPad Prism (San Diego, CA). Analysis of variance (ANOVA) with Bonferroni's multiple comparison test was used to compare the various groups. $P<0.05$ was considered significant.

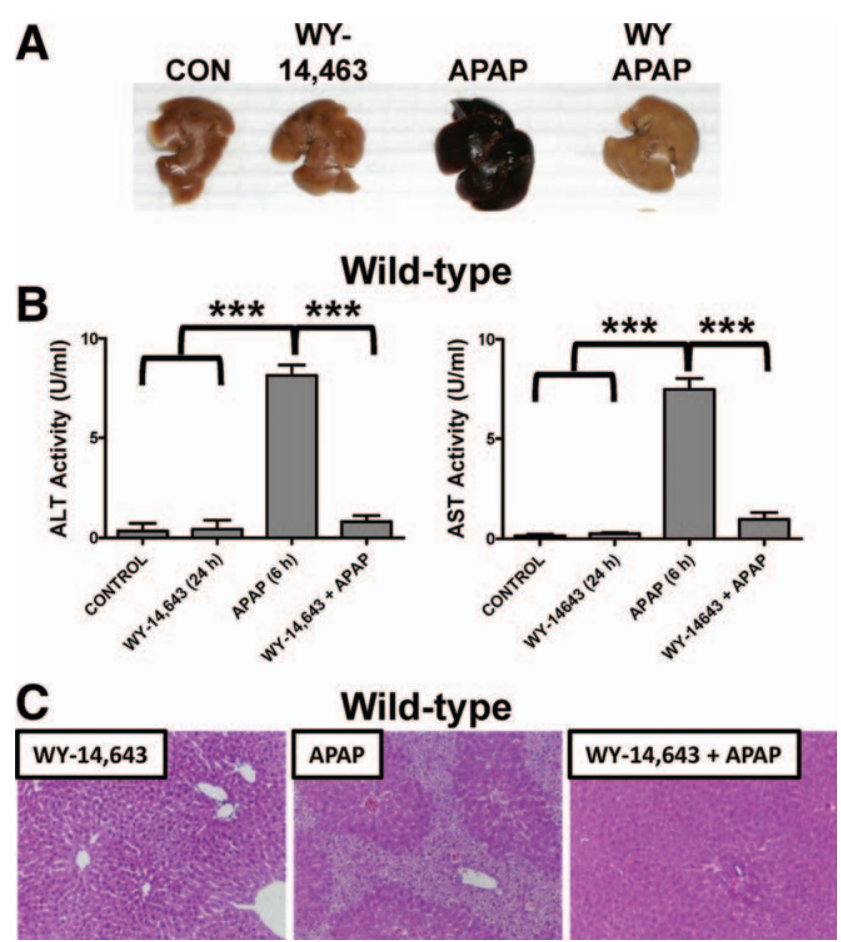

Fig. 1. Wy-14,643 protects against APAP-induced liver toxicity. Mice were fed a diet containing $0.1 \%$ Wy-14,643 24 hours prior to treatment with APAP. (A) Representative livers from control, Wy14,643-treated, APAP-treated, and Wy-14,643/APAP-treated wildtype mice. (B) Serum AST and ALT enzyme levels from control, Wy-14,643treated, APAP-treated, and Wy-14,643/APAP-treated wildtype mice. (C) H\&E staining of livers from Wy-14,643-treated, APAP-treated, and Wy-14,643/APAP-treated wildtype mice. ${ }^{* * * P}<0.001$.

\section{Results}

Wy-14,643 Pretreatment Protects Against APAPinduced Hepatotoxicity. Treatment of wildtype mice with APAP for 6 hours results in massive hepatic toxicity as revealed by gross morphology of the liver (Fig. 1A), increased ALT and AST enzyme levels (Fig. 1B), and faint pericentral and periportal $\mathrm{H} \& \mathrm{E}$ staining of liver parenchyma (Fig. 1C). Pretreatment with Wy14,643 for 24 hours before APAP treatment results in total protection against APAP toxicity; Wy-14,643 treated mice had no evidence of liver damage. At 24 hours post-APAP treatment, Wy-14,643-treated mice were still protected, as indicated by reduced ALT enzyme levels and normal liver histology (Supporting Fig. 1). In contrast, Ppara-null mice exhibited no Wy-14,643 protection against APAP toxicity (shown by increased ALT and AST activities), indicating that the protection was PPAR $\alpha$-dependent (Fig. 2A). To demonstrate that the effect was not specific to the experimental ligand $\mathrm{Wy}_{\mathrm{y}} 14,643$ and to mouse PPAR $\alpha$, PPAR $\alpha$-humanized mice (a human PPAR $\alpha$ gene introduced in the Ppara-null background) treated with 




\section{Ppara-null}

B
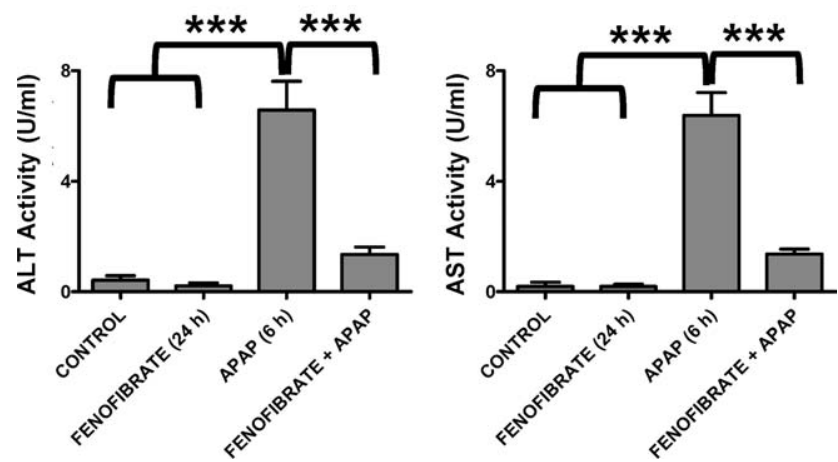

Fig. 2. Wy-14,643 protects against APAP-induced liver toxicity. Mice were fed a diet containing $0.1 \%$ Wy-14,643 24 hours prior to treatment with APAP. (A) Serum AST and ALT enzyme levels from control, Wy-14,643-treated, APAP-treated, and Wy-14,643/APAP-treated Ppara-null mice. (B) Serum AST and ALT enzyme levels from control, fenofibrate-treated, APAP-treated, and fenofibrate/APAP treated hPPAR $^{\text {PAC }}$ mice. ${ }^{* * * P}<0.001$; ns, not significant.

fenofibrate were also protected (Fig. 2B). However, mice treated with the anti-Fas antibody Jo-2 to stimulate Fas receptor-mediated apoptosis were not protected from Wy-14,643 pretreatment (Supporting Fig. 2). Pretreatment with Wy-14,643 did not significantly impact APAP metabolism, as demonstrated by serum profiling of APAP and its metabolites (APAP-NAC, APAP-glucuronide, APAP-CYS) 2 hours after APAP administration (Supporting Fig. 3).

Wy-14,643 Blocks APAP-induced Hepatic Stress Response Genes. In order to understand the transcriptional responses associated with toxic doses of APAPtreatment and potential targets whereby $\operatorname{PPAR} \alpha$ was mediating its protective affects, microarray analysis was carried out on liver mRNA from 6 hours APAPtreated and Wy-14,643-pretreated/APAP-treated mice. A total of 53 genes were up-regulated by APAP and 45 genes up-regulated by Wy-14,643 /APAP; 14 genes were up-regulated by both treatments (greater than 10fold). Most interesting was the marked induction and suppression of $\mathrm{c}$-fos and c-jun expression upon APAP treatment and Wy-14,643-pretreatment prior to APAP administration, respectively (Fig. 3A). qPCR analysis confirmed that c-fos and c-jun mRNAs were robustly induced by APAP and suppressed by Wy-14,643-pretreatment prior to APAP (Fig. 3B). Wy-14,643-pretreatment also blocked APAP-induced phosphorylation of JNK, an important signaling component of APAPinduced toxicity. ${ }^{20-22}$ Wildtype mice treated with APAP for 6 hours exhibited increased p-JNK that was not found with Wy-14,643-pretreatment, whereas Ppara-null mice have increased p-JNK following Wy-14,643-pretreatment (Fig. 3C). To ensure that p-JNK was associated with increased activity, kinase assays were performed and increased p-JNK levels were indeed consistent with elevated p-c-jun levels (Fig. 3C, bottom panel).

Wy-14,643 Blocks APAP-Induced Oxidative Stress. APAP treatment results in a decrease in hepatic levels of GSH at 2 hours and 6 hours, due in part to the production of the quinone NAPQI from APAP that is rapidly neutralized by GSH conjugation by glutathione $S$-transferase. This decrease was partially restored by Wy-14,643-pretreatment. However, the maintenance of GSH levels was even more pronounced in isolated mitochondria (Fig. 4A). $\mathrm{H}_{2} \mathrm{O}_{2}$ levels are inversely correlated with GSH levels and reflect increase oxidative stress. Indeed, Wy-14,643-pretreatment decreased $\mathrm{H}_{2} \mathrm{O}_{2}$ levels elevated by APAP treatment, and this was most pronounced in isolated mitochondria (Fig. 4B).

APAP toxicity is also associated with increased levels of long-chain acylcarnitines in serum that are likely due to mitochondrial damage. ${ }^{15}$ Metabolomics comparison of serum revealed marked differences in serum metabolites between APAP-treated and Wy-14,643pretreatment/APAP as indicated by the scores plot separation of the two groups (Supporting Fig. 4A). This difference was driven, among others, by differences in levels of palmitoylcarnitine that were elevated in APAP-treated mouse serum and normal in Wy-14,643-pretreatment/APAP (Supporting Fig. 4B). Pretreatment with Wy-14,643 prior to APAP administration blocks the increase in palmitoylcarnitines, as indicated by direct quantification of palmitoylcarnitine (Fig. 4C).

At 2 hours post-APAP treatment, both APAP- and Wy-14,643/APAP-treated mice exhibited extensive GSH depletion in both the liver and mitochondria (Fig. 4D).

Role of UCP2 in Wy-14-643 Protection Against APAP-Induced Hepatotoxicity. Because APAP toxicity results in elevated mitochondrial oxidative stress and mitochondrial damage, a role for UCP2 in Wy-14,643 
A

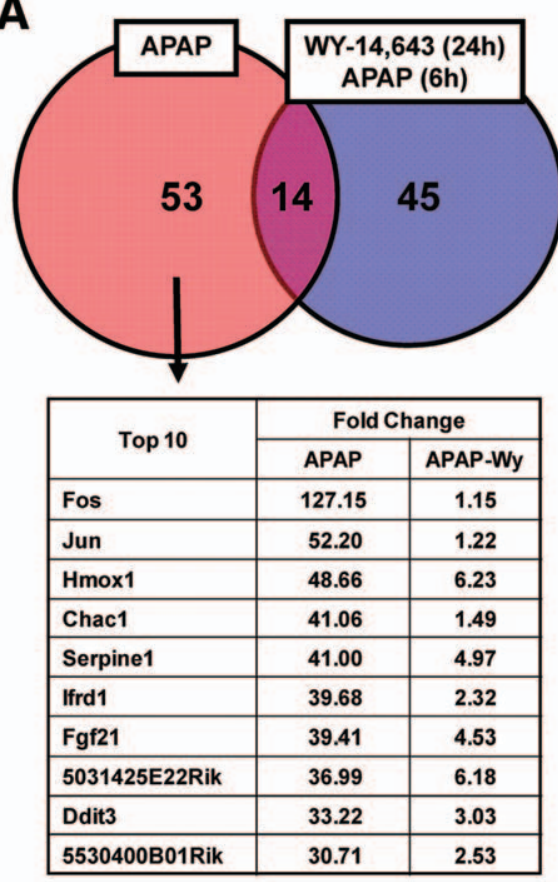

B
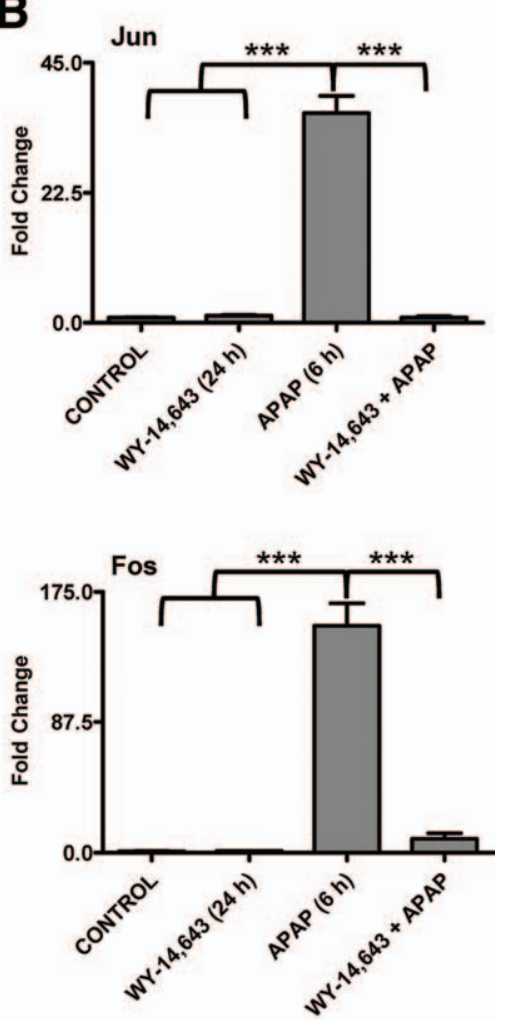

Fig. 3. Changes in gene expression profiles, $\mathrm{c}$-jun and c-fos mRNA levels, and p-JNK associated with Wy-14,643 protection against APAP-induced liver toxicity. (A) Microarray analysis Venn diagram and table of top hits (APAPspecific induction) comparing APAP and Wy14,643/APAP-treated wildtype mice. (B) Analysis of $c$-jun and $c$-fos mRNAs by qPCR in livers from control, Wy-14,643-treated, APAP-treated, and Wy-14,643/APAP-treated wildtype mice. (C) Levels of $\mathrm{p}$-JNK, JNK, and p-C-jun proteins in livers from control, Wy-14,643-treated, APAP-treated, and Wy-14,643/APAP-treated wildtype mice. $* * * P<0.001$

\section{C} Wild-type Ppara-null

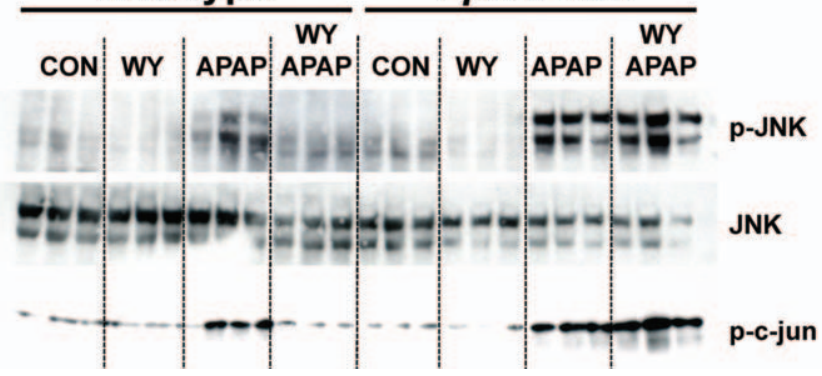

protection against APAP-induced hepatic damage was investigated. UCPs are located in the mitochondrial inner membrane and are associated with decreased hepatic ROS. ${ }^{23,24}$ Wy-14,643 treatment induced UCP2 mRNA in the absence and presence of APAP (Fig. 5A, left panel); similar induction was not observed in Ppara-null mice. Protein levels of UCP2 were also measured in mitochondrial extracts from control and mice treated with $\mathrm{Wy}-14,643$ for 24 hours (Supporting Fig. 5). To determine whether UCP2 has a role in Wy-14,643 protection against APAP hepatotoxicity, Ucp2-null mice were subjected to $\mathrm{Wy}_{\mathrm{y}} 14,643$ and APAP treatment. Mice lacking expression of UCP2 were not protected against APAP-induced toxicity following Wy-14,643, as revealed by serum ALT and AST enzyme levels (Fig. 5B) and liver histology (Fig. 5C). The Ucp2-null mice were still responsive to Wy14,643 , as indicated by induction of the PPAR $\alpha$ target genes Mcad, Cpt1, and Pdk4 (Fig. 6A), indicating that the loss of the protective effects of Wy-14,643 in this model is due to the lack of UCP2 and not PPAR $\alpha$ itself or other PPAR $\alpha$ target genes. Wy-14,643 administration to $U_{c p} 2$-null mice also did not restore mitochondrial GSH loss upon APAP treatment (Fig. 6B) nor fully suppressed the increase in p-JNK (Fig. 6C). These results suggest that the PPAR $\alpha$ target gene UCP2 may be responsible for the protective effect of $\operatorname{PPAR} \alpha$ activators against APAP-induced toxicity.

Ucp2-null mice could have subtle changes that render them resistant to the $\mathrm{Wy}_{\mathrm{y}} 14,643$ protection that are unrelated to UCP2. In order to further establish a role for UCP2 in $\mathrm{Wy}$-14,643-induced protection against APAP hepatotoxicity, forced expression of the protein in livers of wildtype mice was carried out. Recombinant adenoviruses expressing UCP2 (Ad$U_{c p 2}$ ) were constructed and infused into the mouse 


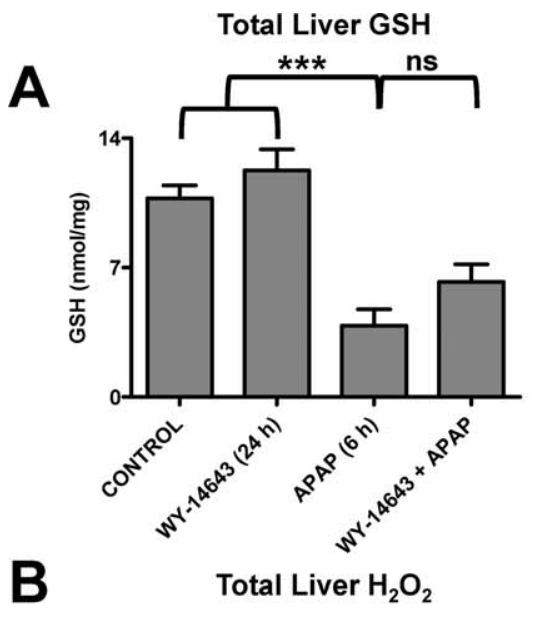

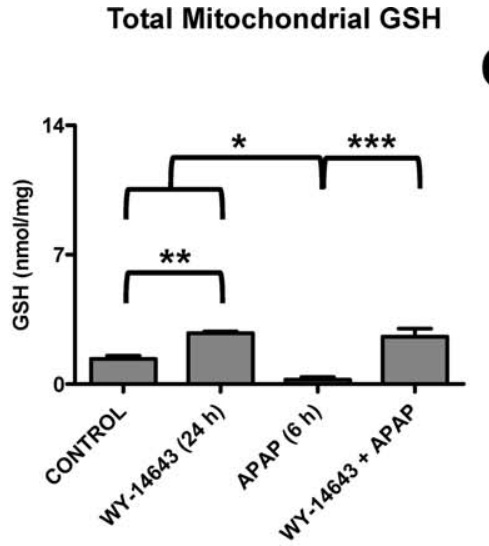

Total Mitochondrial $\mathrm{H}_{2} \mathrm{O}_{2}$



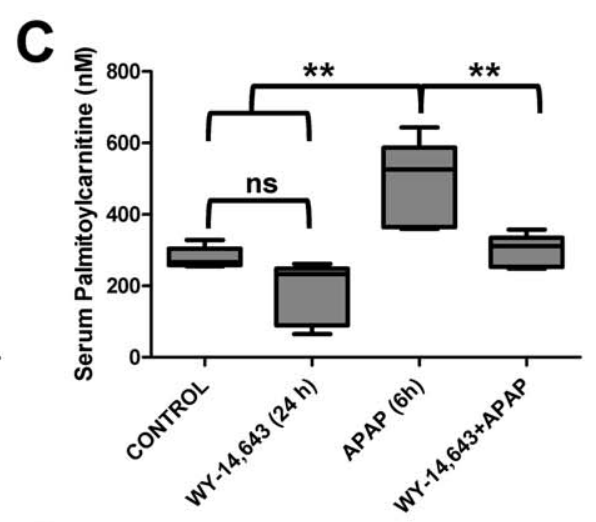

D

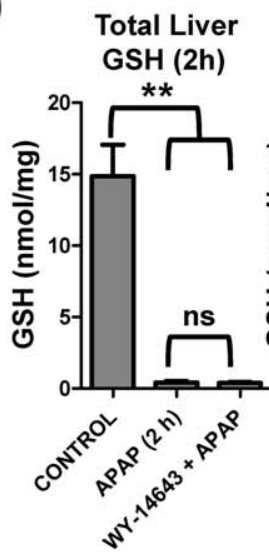

Total Mitochondrial GSH (2h)

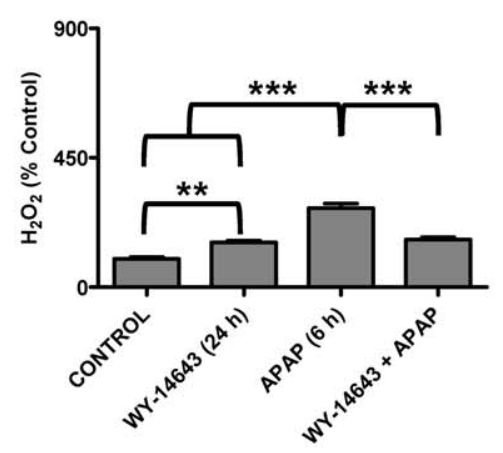

Fig. 4. Changes in oxidative stress markers and acycarnitines associated with Wy-14,643 protection against APAP-induced liver toxicity. (A) Levels of total and mitochondria GSH in livers from control, Wy-14,643-treated, APAP-treated, and Wy-14,643/APAP-treated wildtype mice. (B) Levels of total and mitochondrial $\mathrm{H}_{2} \mathrm{O}_{2}$ in livers from control, Wy-14,643-treated, APAP-treated, and Wy-14,643/APAP-treated wildtype mice. (C) Levels of serum palmitoylcarnitine from control, Wy-14,643-treated, APAP-treated, and Wy-14,643/APAP-treated wildtype mice. (D) Total (left panel) and mitochondrial (right panel) GSH levels were measured in livers from control, APAP-treated, and Wy-14,643/APAP-treated (2 hours after APAP treatment). ${ }^{*} P<0.05, * * P<0.01 ;$ ns, not significant.

livers prior to administration of APAP; UCP2 protein was robustly expressed in the livers of wildtype mice (Fig. 7, bottom right panel). Mice receiving the AdUcp2 were protected against APAP-induced liver toxicity as revealed by $\mathrm{H} \& \mathrm{E}$ staining showing protection against liver necrosis (Fig. 7), lower serum AST and ALT enzyme activities (Fig. 8A), increased mitochondrial GSH (Fig. 8B), and lower p-JNK levels (Fig. $8 \mathrm{C})$. This protection by $\mathrm{Ad}-U_{c p} 2$ was evident at 24 hours post-APAP treatment as indicated by reduced levels of ALT enzyme (Fig. 8D). No protection was found when the control adenovirus expressing Cre recombinase was used and adenovirus itself did not appear to influence CYP2E1 expression (Supporting Fig. 6). ALT activity values for Ad-CrelAPAP-treated mice ranged from 1.8 to $8.0 \mathrm{U} / \mathrm{mL}$, whereas values from Ad-Ucp2/APAPtreated mice ranged from 0.06 to $0.7 \mathrm{U} / \mathrm{mL}$. Even the highest Ad-Ucp2/APAP ALT activity value was still 2.6 times lower than the lowest Ad-CrelAPAP value. These data suggest that UCP2 can protect against APAP- induced hepatotoxicity and that it is a critical target gene responsible for PPAR $\alpha$-mediated protection during APAP-induced hepatotoxicity.

\section{Discussion}

The present study demonstrates a novel, protective role for PPAR $\alpha$ during APAP-induced hepatotoxicity and sheds mechanistic insight into the importance of UCP2 in mediating these protective effects. When the experimental agonist Wy-14,643 was administered prior to a toxic dose of APAP, wildtype mice were completely protected against hepatotoxicity, as revealed by gross liver morphology, H\&E-stained liver sections showing no significant liver damage, and low serum AST and ALT enzyme levels. In addition, livers from mice pretreated with $\mathrm{Wy}_{\mathrm{y}}$ 14,643 prior to APAP had decreased oxidative stress, as revealed by lower $\mathrm{H}_{2} \mathrm{O}_{2}$ levels and higher GSH levels compared with livers from mice only treated with a toxic dose of APAP at 6 
A Ucp2

Fig. 5. Role of UCP2 in Wy14,643 protection against APAPinduced liver toxicity using Ucp2null mice. (A) qPCR analysis of UCP2 mRNA in livers from control, Wy-14,643-treated, APAP-treated, and Wy-14,643/APAP treated wildtype mice. (B) Serum ALT and AST enzyme levels from control, Wy14,643-treated, APAP-treated, and Wy-14,643/APAP-treated Ucp2-null mice. (C) H\&E staining of livers from Wy-14,643-treated, APAPtreated, and Wy-14,643/APAPtreated Ucp2-null mice. ${ }^{* * P}<$ $0.01, \quad * * * P<0.001 ;$ ns, not significant.

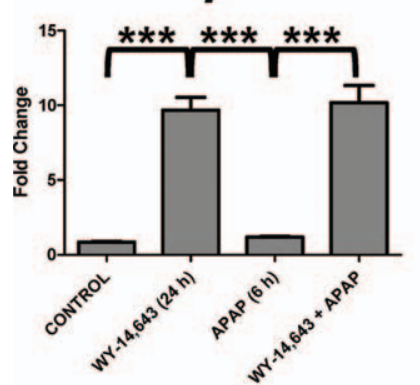

B Ucp2-null
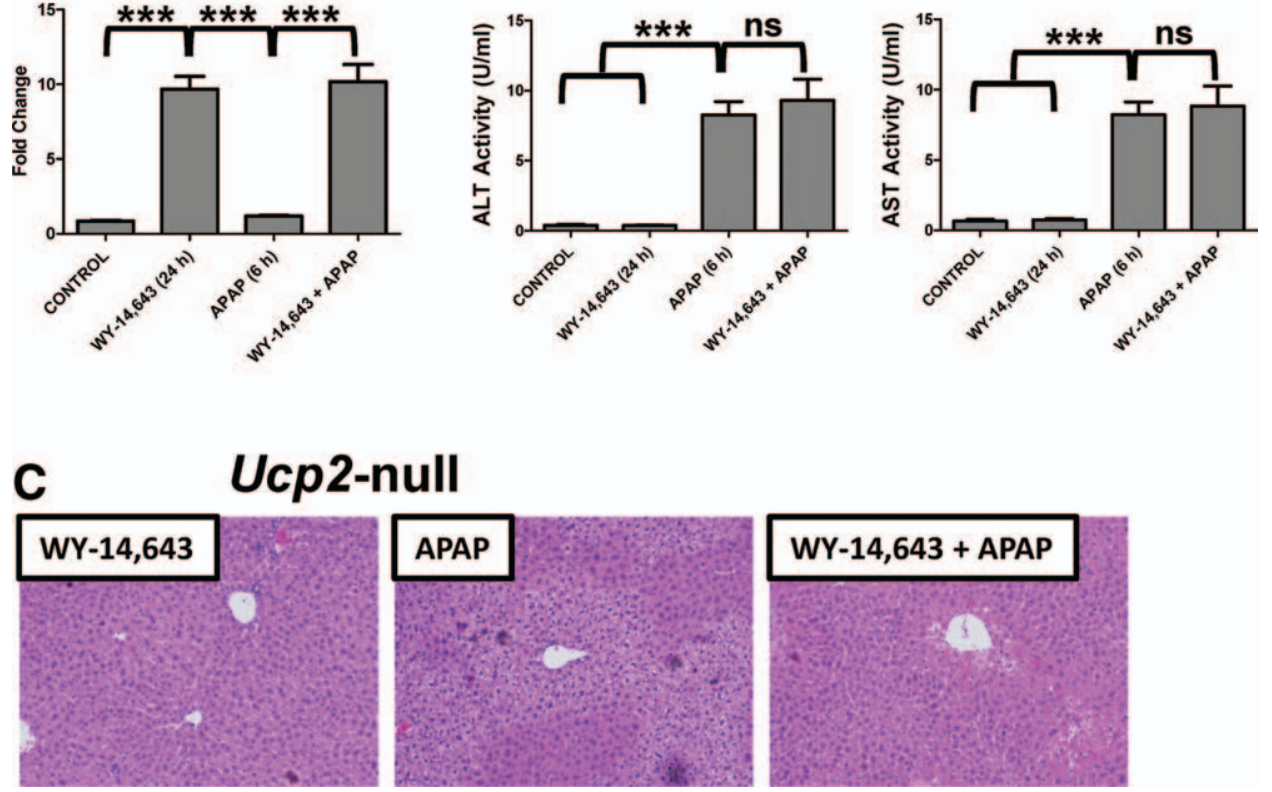

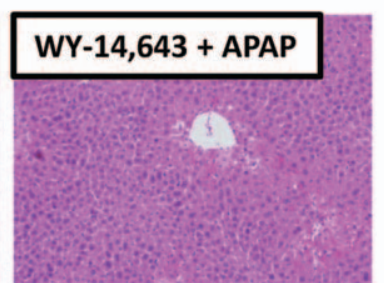

hours. Interestingly, Wy-14,643-treated mice exhibited a rapid reduction of GSH levels at 2 hours post-APAP treatment. In fact, these results are in strong agreement with a previous report demonstrating that mice lacking CYP2E1 were protected from APAP-induced hepatotoxicity due in part to activation of PPAR $\alpha .{ }^{15}$ Evidence for decreased oxidative stress was also revealed by reduced induction of c-jun and c-fos and lower
p-JNK levels upon Wy-14,643 pretreatment. This effect was specific to APAP-induced hepatotoxicity and JNK pathway attenuation, as Jo-2 treatment that stimulates the Fas death pathway was unaffected by pretreatment with $\mathrm{Wy}_{\mathrm{y}} \mathrm{14}, 643$. This observation is consistent with a previous report demonstrating that attenuating JNK signaling did not protect from Fasmediated cell death. ${ }^{20}$ In general, it is the enhanced
A

Ucp2-null
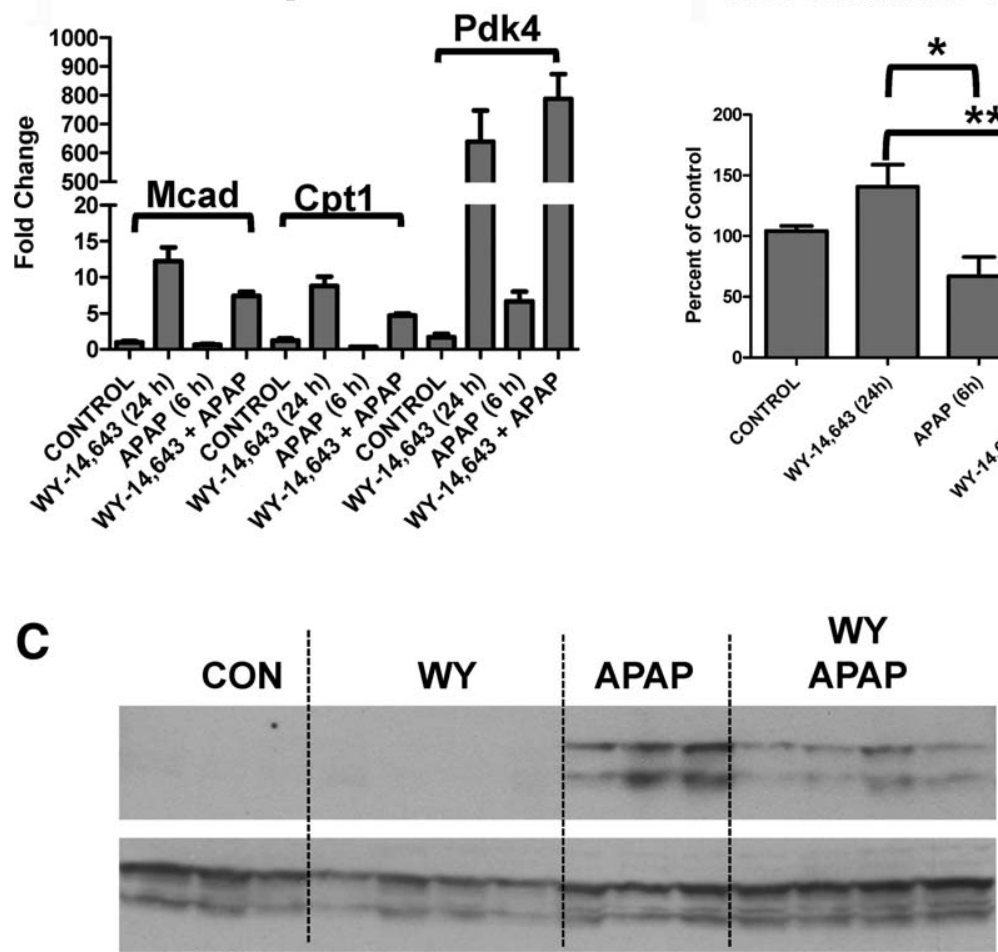

B Mitochondrial GSH



Fig. 6. Role of UCP2 in Wy-14,643 protection against APAP-induced liver toxicity using Ucp2null mice. (A) qPCR analysis of MCAD, CPT1, and PDK4 mRNA in livers from control, Wy14,643-treated, APAP-treated, and Wy-14,643/ APAP-treated Ucp2-null mice. (B) Levels of mitop-JNK chondrial GSH control, Wy-14,643-treated, APAPtreated, and Wy-14,643/APAP-treated Ucp2-null mice. (C) Levels of $\mathrm{p}$-JNK, JNK, and p-c-jun proteins in livers from control, Wy-14,643-treated, APAP-treated, and Wy-14,643/APAP-treated Ucp2-null mice. ${ }^{*} P<0.05,{ }^{* *} P<0.01$. 



Fig. 7. Role of UCP2 in Wy-14,643 protection against APAP-induced liver toxicity using forced expression of UCP2 in livers of wildtype mice. H\&E staining of livers from wildtype mice administered Ad-Ucp2 and Ad-Cre prior to APAP treatment. Western blot of UCP2 protein in Ad-Ucp2 and Ad-Cre treated mice. Uptake of virus was monitored by PCR of Cre recombinase cDNA.

and persistent PPAR $\alpha$ activation prior to APAP treatment that is important for mediating these effects. However, studies examining the role of PPAR $\alpha$ activation post-APAP treatment should be conducted to determine if this pathway holds any promises for therapeutic intervention.

Previous studies revealed that acylcarnitines were elevated early after APAP treatment and that their elevation was indicative of mitochondrial damage and dysfunction. ${ }^{15,19}$ In the present study these observations were confirmed, as palmitoylcarnitine was elevated by toxic doses of APAP and maintained at normal levels (compared with untreated controls) by Wy-14,643 pretreatment. The enhanced toxicity in the Ppara-null mice revealed that the protective response to Wy14,643 was PPAR $\alpha$-dependent. Protection of APAP toxicity by Wy-14,643 also extended to human PPAR $\alpha$, as indicated by similar protection from APAPinduced hepatotoxicity in PPAR $\alpha$-humanized mice receiving the PPAR $\alpha$ activator fenofibrate.

PPAR $\alpha$ activates a large number of target genes primarily associated with fatty acid transport and catabolism. Thus, it was important to determine which among these target genes afforded protection. Earlier studies revealed that among the earliest events associated with APAP toxicity was elevated oxidative stress as a result of oxidation of APAP to the quinone metabolite NAPQI by cytochromes P450, notably by
Fig. 8. Role of UCP2 in Wy14,643 protection against APAPinduced liver toxicity using forced expression of UCP2 in livers of wildtype mice. (A) Effect of AdUcp2 and Ad-Cre prior to APAP treatment on serum ALT and AST enzyme levels. (B) Effect of AdUcp2 and Ad-Cre prior to APAP treatment on total mitochondrial GSH levels in livers of wildtype mice. (C) Effect of Ad-Ucp2 and Ad-Cre prior to APAP treatment on JNK and $p$-JNK protein levels in livers of wildtype mice. (D) ALT enzyme levels in APAP and AdUcp2 treated mice after 24 hours APAP treatment. ${ }^{*} P<0.05, * * P$ $<0.01$.
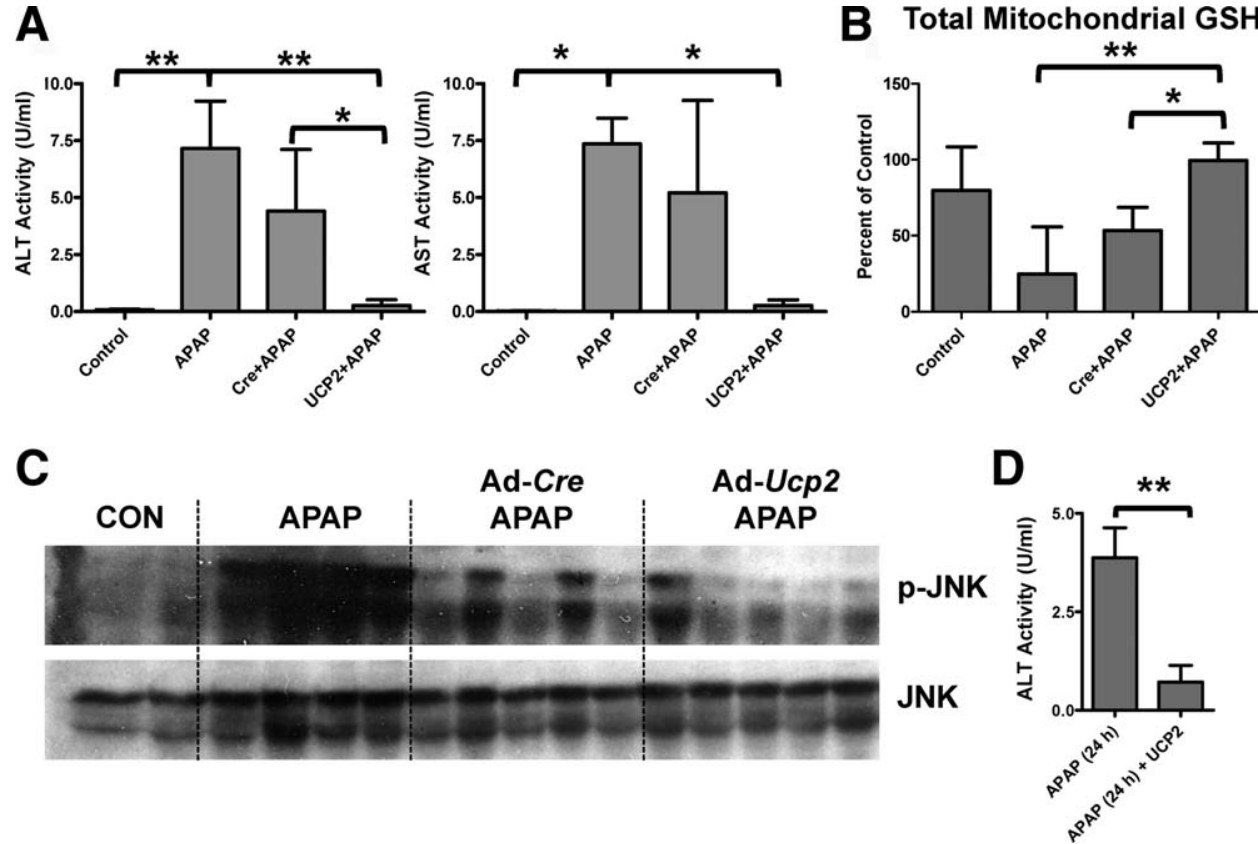
CYP2E1, ${ }^{11,25}$ and dramatic reduction of cellular antioxidants including GSH. This is likely followed by mitochondrial damage leading to cell death and these effects may be partially mediated by reduced PPAR $\alpha$ activity in the presence of high doses of APAP. ${ }^{15,19}$ The findings of decreased oxidative stress with Wy14,643 suggest that a target gene that influences liver ROS and/or preserves mitochondrial fatty acid $\beta$-oxidation might be a PPAR $\alpha$-dependent candidate responsible for the protective effects from APAP-induced hepatotoxicity.

UCPs are a small family of transporters present in the inner mitochondria membrane that have been implicated in the protection against ROS generation in macrophages. Many studies have revealed that UCPs regulate mitochondrial $\mathrm{ROS}^{26}$ and, as in the case of UCP2, can be activated by increased levels of fatty acids $^{27}$ such as arachidonate. Furthermore, UCP2 has been reported to be important for promoting fatty acid $\beta$-oxidation, ${ }^{28}$ which under conditions of APAPinduced inhibition of fatty acid catabolism makes it a likely target for mediating these protective effects. UCP2 up-regulation in liver is also found in pathologic conditions such as steatosis and obesity, where increased or perturbed fatty acid oxidation is observed. $^{27,29}$ High UCP2 expression is also found in certain tumors where it is thought that the tumor cells utilize the GSH preserving qualities of UCP2 to promote growth and reduce ROS levels. UCP2 decreases ROS in liver through a mechanism that is not completely understood, ${ }^{23,24}$ yet the recent report of its crystal structure is an important step in understanding the function of UCP2. ${ }^{30}$ UCP2 may serve a protective role in mitochondria by reducing ROS levels directly, lowering mitochondrial membrane potential, transporting fatty acids and fatty acid peroxides, or a combination of the above. Most important, UCP2 is markedly induced by $\operatorname{PPAR} \alpha$ activation in liver, specifically hepatocytes ${ }^{7}$ coincident with the induction of mitochondrial and peroxisomal enzymes involved in fatty acid $\beta$-oxidation. Based on these data, UCP2 was viewed as a potential candidate for a Wy-14,643-induced protein that could protect from APAP toxicity. Indeed, mice lacking expression of UCP2 were not protected against APAP toxicity by $\mathrm{Wy}-14,643$, whereas forced overexpression of UCP2 in the liver of wildtype mice also protected against APAP-induced toxicity in the absence of Wy-14,643. It is noteworthy, however, that sustained expression of UCP2 may in fact be deleterious, suggesting that UCP2 expression must be tightly controlled in order to maintain its salubrious qualities. Furthermore, other UCP family members, namely
UCP3, may also contribute to the protection given the reduced JNK phosphorylation in Ucp2-null mice treated with WY-14,643. Moreover, it is likely that the combined activities of PPAR $\alpha$ targets facilitate maximal protection, and their roles specifically at the level of maintaining mitochondrial function warrant further investigation.

The question arises whether UCP2 plays a role in protecting the liver against ROS under normal physiological conditions, such as during mitochondrial fatty acid $\beta$-oxidation. In general, Ucp2-null mouse livers have elevated ROS compared with wildtype mice. ${ }^{31}$ Thus, UCP2 could serve as a general protector of the liver and, in particular, of the mitochondria from oxidative stress produced by normal metabolism. Under conditions of fasting, PPAR $\alpha$ is activated by endogenous ligands, resulting in induction of peroxisome and mitochondrial fatty acid oxidation. ${ }^{32}$ Indeed, PPAR $\alpha$ activation by starvation results in elevated mitochondrial ROS. ${ }^{33}$ This results in elevated ROS that is neutralized by the coinduced UCP2. In the case of chemically induced hepatotoxicity that results in massively elevated and lethal levels of ROS, the protective effect of UCPs are even more essential.

Another intriguing possibility was demonstrated in a recent study where the authors reported an intimate relationship between elevated ROS and UCPs. ${ }^{8} \mathrm{UCP} 2$ (and UCP3) contain reactive cysteines that can be modified by GSH. The deglutathionylation/glutathionylation regulates UCP2 and UCP3 activity. In the presence of elevated ROS, GSH is depleted and the proteins lose the conjugated glutathione, thereby rendering them active and able to neutralized ROS. Under the conditions of APAP-induced hepatotoxicity, elevated ROS levels likely mediate similar activation of UCP2, however, only following activation by PPAR $\alpha$.

In conclusion, this study adds to our understanding of how toxic doses of APAP mediate hepatotoxicity and provides new insight into the importance of $\operatorname{PPAR} \alpha$ activation in maintaining proper mitochondrial function, most likely through UCP2 under normal and pathologic conditions. Further, this study lends even greater support for how repression of PPAR $\alpha$ activation can lead to deleterious effects. Using Ucp2null mice and mice transiently expressing UCP2 (from adenovirus), a convincing role for UPC2 in protecting against APAP-induced hepatotoxicity through preservation of mitochondrial function was demonstrated. Further studies to determine the mechanisms by which UCP2 facilitates this protection are warranted and will provide greater understanding by which ROS elevating hepatoxicants, such as APAP, mediate their effects. 
Acknowledgment: We thank Jared Correll and Jessica Montanez for technical assistance and Dr. Chi Chen for insightful discussions.

\section{References}

1. Peters JM, Cattley RC, Gonzalez FJ. Role of PPAR alpha in the mechanism of action of the nongenotoxic carcinogen and peroxisome proliferator Wy-14,643. Carcinogenesis 1997;18:2029-2033.

2. Shah YM, Morimura K, Yang Q, Tanabe T, Takagi M, Gonzalez FJ. Peroxisome proliferator-activated receptor alpha regulates a microRNAmediated signaling cascade responsible for hepatocellular proliferation. Mol Cell Biol 2007;27:4238-4247.

3. Gonzalez FJ, Shah YM. PPARalpha: mechanism of species differences and hepatocarcinogenesis of peroxisome proliferators. Toxicology 2008; 246:2-8.

4. Aoyama T, Peters JM, Iritani N, Nakajima T, Furihata K, Hashimoto $\mathrm{T}$, et al. Altered constitutive expression of fatty acid-metabolizing enzymes in mice lacking the peroxisome proliferator-activated receptor alpha (PPARalpha). J Biol Chem 1998;273:5678-5684.

5. Kersten S, Seydoux J, Peters JM, Gonzalez FJ, Desvergne B, Wahli W. Peroxisome proliferator-activated receptor alpha mediates the adaptive response to fasting. J Clin Invest 1999;103:1489-1498.

6. Mandard S, Muller M, Kersten S. Peroxisome proliferator-activated receptor alpha target genes. Cell Mol Life Sci 2004;61:393-416.

7. Nakatani T, Tsuboyama-Kasaoka N, Takahashi M, Miura S, Ezaki O. Mechanism for peroxisome proliferator-activated receptor-alpha activator-induced up-regulation of UCP2 mRNA in rodent hepatocytes. J Biol Chem 2002;277:9562-9569.

8. Mailloux RJ, Seifert EL, Bouillaud F, Aguer C, Collins S, Harper ME. Glutathionylation Acts as a Control Switch for Uncoupling Proteins UCP2 and UCP3. J Biol Chem 2011;286:21865-21875.

9. D’Arcy PF. Paracetamol. Adverse Drug React Toxicol Rev 1997;16: 9-14.

10. Mazer M, Perrone J. Acetaminophen-induced nephrotoxicity: pathophysiology, clinical manifestations, and management. J Med Toxicol 2008;4:2-6.

11. Gonzalez FJ. The 2006 Bernard B. Brodie Award Lecture. Cyp2e1. Drug Metab Dispos 2007;35:1-8.

12. Manautou JE, Hoivik DJ, Tveit A, Hart SG, Khairallah EA, Cohen SD. Clofibrate pretreatment diminishes acetaminophen's selective covalent binding and hepatotoxicity. Toxicol Appl Pharmacol 1994;129: 252-263.

13. Manautou JE, Tveit A, Hoivik DJ, Khairallah EA, Cohen SD. Protection by clofibrate against acetaminophen hepatotoxicity in male CD-1 mice is associated with an early increase in biliary concentration of acetaminophen-glutathione adducts. Toxicol Appl Pharmacol 1996;140: 30-38.

14. Chen C, Hennig GE, Whiteley HE, Corton JC, Manautou JE. Peroxisome proliferator-activated receptor alpha-null mice lack resistance to acetaminophen hepatotoxicity following clofibrate exposure. Toxicol Sci 2000;57:338-344.

15. Chen C, Krausz KW, Shah YM, Idle JR, Gonzalez FJ. Serum metabolomics reveals irreversible inhibition of fatty acid beta-oxidation through the suppression of PPARalpha activation as a contributing mechanism of acetaminophen-induced hepatotoxicity. Chem Res Toxicol 2009;22:699-707.
16. Lee SS, Pineau T, Drago J, Lee EJ, Owens JW, Kroetz DL, et al. Targeted disruption of the alpha isoform of the peroxisome proliferatoractivated receptor gene in mice results in abolishment of the pleiotropic effects of peroxisome proliferators. Mol Cell Biol 1995;15:3012-3022.

17. Yang Q, Nagano T, Shah Y, Cheung C, Ito S, Gonzalez FJ. The PPAR alpha-humanized mouse: a model to investigate species differences in liver toxicity mediated by PPAR alpha. Toxicol Sci 2008;101:132-139.

18. Hong Y, Fink BD, Dillon JS, Sivitz WI. Effects of adenoviral overexpression of uncoupling protein-2 and -3 on mitochondrial respiration in insulinoma cells. Endocrinology 2001;142:249-256.

19. Chen C, Krausz KW, Idle JR, Gonzalez FJ. Identification of novel toxicity-associated metabolites by metabolomics and mass isotopomer analysis of acetaminophen metabolism in wild-type and Cyp2e1-null mice. J Biol Chem 2008;283:4543-4559.

20. Henderson NC, Pollock KJ, Frew J, Mackinnon AC, Flavell RA, Davis $\mathrm{RJ}$, et al. Critical role of c-jun (NH2) terminal kinase in paracetamolinduced acute liver failure. Gut 2007;56:982-990.

21. Hanawa N, Shinohara M, Saberi B, Gaarde WA, Han D, Kaplowitz N. Role of JNK translocation to mitochondria leading to inhibition of mitochondria bioenergetics in acetaminophen-induced liver injury. J Biol Chem 2008;283:13565-13577.

22. Win S, Than TA, Han D, Petrovic LM, Kaplowitz N. c-Jun N-terminal kinase (JNK)-dependent acute liver injury from acetaminophen or tumor necrosis factor (TNF) requires mitochondrial Sab protein expression in mice. J Biol Chem 2011;286:35071-35078.

23. Nedergaard J, Cannon B. The 'novel uncoupling' proteins UCP2 and UCP3: what do they really do? Pros and cons for suggested functions. Exp Physiol 2003;88:65-84.

24. Brand MD, Esteves TC. Physiological functions of the mitochondrial uncoupling proteins UCP2 and UCP3. Cell Metab 2005;2:85-93.

25. Zaher H, Buters JTM, Ward JM, Bruno MK, Lucas AM, Stern ST, et al. Protection against acetaminophen toxicity in CYP1A2 and CYP2E1 double-null mice. Toxicol Appl Pharmacol 1998;152: 193-199.

26. Sluse FE, Jarmuszkiewicz W, Navet R, Douette P, Mathy G, Sluse-Goffart CM. Mitochondrial UCPs: new insights into regulation and impact. Biochim Biophys Acta Bioenerg 2006;1757:480-485.

27. Yonezawa T, Kurata R, Hosomichi K, Kono A, Kimura M, Inoko H. Nutritional and hormonal regulation of uncoupling protein 2. IUBMB Life 2009;61:1123-1131.

28. Pecqueur C, Bui T, Gelly C, Hauchard J, Barbot C, Bouillaud F, et al. Uncoupling protein-2 controls proliferation by promoting fatty acid oxidation and limiting glycolysis-derived pyruvate utilization. FASEB J 2008;22:9-18.

29. Sluse FE, Jarmuszkiewicz W, Navet R, Douette P, Mathy G, Sluse-Goffart CM. Mitochondrial UCPs: new insights into regulation and impact. Biochim Biophys Acta 2006;1757:480-485.

30. Berardi MJ, Shih WM, Harrison SC, Chou JJ. Mitochondrial uncoupling protein 2 structure determined by NMR molecular fragment searching. Nature 2011;476:109-113.

31. Kuhla A, Hettwer C, Menger MD, Vollmar B. Oxidative stress-associated rise of hepatic protein glycation increases inflammatory liver injury in uncoupling protein-2 deficient mice. Lab Invest 2010;90:1189-1198.

32. Kersten S, Seydoux J, Peters JM, Gonzalez FJ, Desvergne B, Wrahli W. Peroxisome proliferator-activated receptor alpha mediates the adaptive response to fasting. J Clin Invest 1999;103:1489-1498.

33. Abdelmegeed MA, Moon KH, Hardwick JP, Gonzalez FJ, Song BJ. Role of peroxisome proliferator-activated receptor-alpha in fastingmediated oxidative stress. Free Radic Biol Med 2009;47:767-778. 